\title{
A stability property for coefficients in Kronecker products of complex $S_{n}$ characters
}

\author{
Ernesto Vallejo* \\ Universidad Nacional Autónoma de México \\ Instituto de Matemáticas, Unidad Morelia \\ Apartado Postal 61-3, Xangari \\ 58089 Morelia, Mich., MEXICO \\ vallejo@matmor . unam.mx
}

Submitted: Apr 29, 2009; Accepted: Jun 12, 2009; Published: Jul 2, 2009

Mathematics Subject Classification: 05E10

\begin{abstract}
In this note we make explicit a stability property for Kronecker coefficients that is implicit in a theorem of Y. Dvir. Even in the simplest nontrivial case this property was overlooked despite of the work of several authors. As applications we give a new vanishing result and a new formula for some Kronecker coefficients.
\end{abstract}

\section{Introduction}

Let $\lambda, \mu, \nu$ be partitions of a positive integer $m$ and let $\chi^{\lambda}, \chi^{\mu}, \chi^{\nu}$ be their corresponding complex irreducible characters of the symmetric group $S_{m}$. It is a long standing problem to give a satisfactory method for computing the multiplicity

$$
\mathrm{k}(\lambda, \mu, \nu):=\left\langle\chi^{\lambda} \otimes \chi^{\mu}, \chi^{\nu}\right\rangle
$$

of $\chi^{\nu}$ in the Kronecker product $\chi^{\lambda} \otimes \chi^{\mu}$ of $\chi^{\lambda}$ and $\chi^{\mu}$ (here $\langle\cdot, \cdot \cdot\rangle$ denotes the inner product of complex characters). Via the Frobenius map, $\mathrm{k}(\lambda, \mu, \nu)$ is equal to the multiplicity of the Schur function $s_{\nu}$ in the internal product of Schur functions $s_{\lambda} * s_{\mu}$, namely

$$
\mathrm{k}(\lambda, \mu, \nu)=\left\langle s_{\lambda} * s_{\mu}, s_{\nu}\right\rangle,
$$

where $\langle\cdot, \cdot\rangle$ denotes the scalar product of symmetric functions.

The first stability property for Kronecker coefficients was observed by F. Murnaghan without proof in [8]. This property can be stated in the following way: Let $\bar{\lambda}, \bar{\mu}, \bar{\nu}$

*Supported by CONACYT-Mexico, 47086-F and UNAM-DGAPA IN103508 
be partitions of $a, b, c$, respectively. Define $\lambda(n):=(n-a, \bar{\lambda}), \mu(n):=(n-b, \bar{\mu})$, $\nu(n):=(n-c, \bar{\nu})$. Then the coefficient $\mathrm{k}(\lambda(n), \mu(n), \nu(n))$ is constant for all $n$ bigger than some integer $N(\bar{\lambda}, \bar{\mu}, \bar{\nu})$. Complete proofs of this property were given by M. Brion [3] using algebraic geometry and E. Vallejo [13] using combinatorics of Young tableaux. Both proofs give different lower bounds $N(\bar{\lambda}, \bar{\mu}, \bar{\nu})$ for the stability of $\mathrm{k}(\lambda(n), \mu(n), \nu(n))$, for all partitions $\bar{\lambda}, \bar{\mu}, \bar{\nu}$. C. Ballantine and R. Orellana [1] gave an improvement of one of these lower bounds for a particular case.

Here we make explicit another stability property for Kronecker coefficients that is implicit in the work of Y. Dvir (Theorem 2.4' in [5]). This property can be stated as follows: Let $p, q$ and $r$ be positive integers such that $p=q r$. Let $\lambda=\left(\lambda_{1}, \ldots, \lambda_{p}\right)$, $\mu=\left(\mu_{1}, \ldots, \mu_{q}\right), \nu=\left(\nu_{1}, \ldots, \nu_{r}\right)$ be partitions of some nonnegative integer $m$ satisfying $\ell(\lambda) \leq p, \ell(\mu) \leq q, \ell(\nu) \leq r$, that is, some parts of $\lambda, \mu$ and $\nu$ could be zero. For any positive integers $t$ and $n$ let $(t)^{n}$ denote the vector $(t, \ldots, t) \in \mathbb{N}^{n}$; and for any partition $\lambda=\left(\lambda_{1}, \ldots, \lambda_{p}\right)$ of length at most $p$ let $\lambda+(t)^{p}$ denote the partition $\left(\lambda_{1}+t, \ldots, \lambda_{p}+t\right)$. Then we have

Theorem 3.1. With the above notation

$$
\mathrm{k}(\lambda, \mu, \nu)=\mathrm{k}\left(\lambda+(t)^{p}, \mu+(r t)^{q}, \nu+(q t)^{r}\right) .
$$

It should be noted that even in the simplest nontrivial case, when $q=2=r$ and $p=4$, this property was overlooked despite of the work of several authors $[1,2,9,10]$. In this situation Remmel and Whitehead noticed (Theorems 3.1 and 3.2 in [9]) that the coefficient $\mathrm{k}(\lambda, \mu, \nu)$ has a much simpler formula if $\lambda_{3}=\lambda_{4}$. The main theorem provides an explanation for that. We also obtain a new formula for $\mathrm{k}(\lambda, \mu, \nu)$ in this case.

This note is organized as follows. Section 2 contains the definitions and notation about partitions needed in this paper. In Section 3 we give the proof of the main theorem. Section 4 deals with the Kronecker coefficient $\mathrm{k}(\lambda, \mu, \nu)$ when $\ell(\lambda)=\ell(\mu) \ell(\nu)$. In particular, we give, in this case, a new vanishing condition. Finally, in Section 5 we give an application of the main theorem.

\section{Partitions}

In this section we recall the notation about partitions needed in this paper. See for example [6, 7, 11, 12].

For any nonnegative integer $n$ let $[n]:=\{1, \ldots, n\}$. A partition is a vector $\lambda=$ $\left(\lambda_{1}, \ldots, \lambda_{p}\right)$ of nonnegative integers arranged in decreasing order $\lambda_{1} \geq \cdots \geq \lambda_{p}$. We consider two partitions equal if they differ by a string of zeros at the end. For example $(3,2,1)$ and $(3,2,1,0,0)$ represent the same partition. The length of $\lambda$, denoted by $\ell(\lambda)$, is the number of positive parts of $\lambda$. The size of $\lambda$, denoted by $|\lambda|$, is the sum of its parts; if $|\lambda|=m$, we say that $\lambda$ is a partition of $m$ and denote it by $\lambda \vdash m$. The partition conjugate to $\lambda$ is denoted by $\lambda^{\prime}$. A composition of $m$ is a vector $\pi=\left(\pi_{1}, \ldots, \pi_{r}\right)$ of positive integers such that $\sum_{i=1}^{r} \pi_{i}=m$. 
The diagram of $\lambda=\left(\lambda_{1}, \ldots, \lambda_{p}\right)$, also denoted by $\lambda$, is the set of pairs of integers

$$
\lambda=\left\{(i, j) \mid i \in[p], j \in\left[\lambda_{i}\right]\right\} .
$$

The identification of $\lambda$ with its diagram permits us to use set theoretic notation for partitions. If $\delta$ is another partition and $\delta \subseteq \lambda$, we denote by $\lambda / \delta$ the skew diagram consisting of the pairs in $\lambda$ that are not in $\delta$, and by $|\lambda / \delta|$ its cardinality. If $\mu$ is another partition, then $\lambda \cap \mu$ denotes the set theoretic intersection of $\lambda$ and $\mu$.

\section{Main theorem}

3.1 Theorem. Let $\lambda, \mu, \nu$ be partitions of some integer $m$. Let $p, q, r$ be integers such that $p \geq \ell(\lambda), q \geq \ell(\mu), r \geq \ell(\nu)$ and $p=q r$. Then for any positive integer $t$ we have

$$
\mathrm{k}(\lambda, \mu, \nu)=\mathrm{k}\left(\lambda+(t)^{p}, \mu+(r t)^{q}, \nu+(q t)^{r}\right) .
$$

The proof of the main theorem will follow from Dvir's theorem

3.2 Theorem. [5, Theorem 2.4'] Let $\lambda, \mu, \nu$ be partitions of $n$ such that $\ell(\nu)=\left|\lambda \cap \mu^{\prime}\right|$. Let $l=\ell(\nu)$ and $\rho=\nu-\left(1^{l}\right)$. Then

$$
\mathrm{k}(\lambda, \mu, \nu)=\left\langle\chi^{\lambda / \lambda \cap \mu^{\prime}} \otimes \chi^{\mu / \lambda^{\prime} \cap \mu}, \chi^{\rho}\right\rangle .
$$

Proof of theorem 3.1. It is enough to prove the theorem for $t=1$. The general case follows by repeated application of the particular case. Let $\alpha=\lambda+(1)^{p}, \beta=\mu+(r)^{q}$ and $\gamma=\nu+(q)^{r}$. Then $\beta \cap \gamma^{\prime}=(r)^{q}$. In particular, $\left|\beta \cap \gamma^{\prime}\right|=p=\ell(\alpha)$. So, we have $\beta / \beta \cap \gamma^{\prime}=\mu$ and $\gamma / \beta^{\prime} \cap \gamma=\nu$. Thus, by Dvir's theorem, we have

$$
\mathrm{k}(\beta, \gamma, \alpha)=\mathrm{k}(\mu, \nu, \lambda) .
$$

The claim follows from the symmetry $\mathrm{k}(\lambda, \mu, \nu)=\mathrm{k}(\mu, \nu, \lambda)$ of Kronecker coefficients.

3.3 Example. To illustrate how Dvir's theorem applies, let $\lambda=(8,4), \mu=(6,6)$ and $\nu=(5,3,2,2)$. Then $\lambda \cap \mu^{\prime}=(2,2)=\lambda^{\prime} \cap \mu, \lambda / \lambda \cap \mu^{\prime}=(6,2), \mu / \lambda^{\prime} \cap \mu=(4,4)$ and $\nu-\left(1^{4}\right)=(4,2,1,1)$. After two applications of Dvir's theorem we get

$$
\begin{aligned}
\mathrm{k}((8,4),(6,6),(5,3,2,2)) & =\mathrm{k}((6,2),(4,4),(4,2,1,1)) \\
& =\mathrm{k}((4),(2,2),(3,1))=0 .
\end{aligned}
$$

\section{The case $\ell(\lambda)=\ell(\mu) \ell(\nu)$}

In this section we give a general result for the Kronecker coefficient $\mathrm{k}(\lambda, \mu, \nu)$ when $\ell(\lambda)=$ $\ell(\mu) \ell(\nu)$. On the one hand it gives a new vanishing condition. On the other hand, when this vanishing condition does not hold, it reduces the computation of $\mathrm{k}(\lambda, \mu, \nu)$ to the computation of a simpler Kronecker coefficient. 
Let $m$ be a positive integer, $\lambda, \mu$ be partitions of $m$ and $\pi=\left(\pi_{1}, \ldots, \pi_{r}\right)$ be a composition of $m$. Let $\rho(i) \vdash \pi_{i}$ for $i \in[r]$. A sequence $T=\left(T_{1}, \ldots, T_{r}\right)$ of tableaux is called a Littlewood-Richardson multitableau of shape $\lambda$, content $(\rho(1), \ldots, \rho(r))$ and type $\pi$ if

(1) there exists a sequence of partitions

$$
\varnothing=\lambda(0) \subset \lambda(1) \subset \cdots \subset \lambda(r)=\lambda
$$

such that $|\lambda(i) / \lambda(i-1)|=\pi_{i}$ for all $i \in[r]$, and

(2) $T_{i}$ is Littlewood-Richardson tableau of shape $\lambda(i) / \lambda(i-1)$ and content $\rho(i)$, for all $i \in[r]$.

For example,

\begin{tabular}{|c|c|c|c|c|c|c|c|c|c|}
\hline 1 & 1 & 1 & 1 & 1 & 1 & 1 & 1 & 1 & 1 \\
\hline 2 & 2 & 2 & 2 & 2 & 2 & 2 & 2 & & \\
\hline 3 & 3 & 2 & 2 & 3 & & & & & \\
\hline 3 & 3 & & & & & & & & \\
\hline
\end{tabular}

is a Littlewood-Richardson multitableau of shape $(10,8,5,2)$, type $(10,8,7)$ and content $((4,4,2),(3,3,2),(3,3,1))$.

Let $\operatorname{LR}(\lambda, \mu ; \pi)$ denote the set of pairs $(S, T)$ of Littlewood-Richardson multitableaux of shape $(\lambda, \mu)$, same content and type $\pi$. This means that $S=\left(S_{1}, \ldots, S_{r}\right)$ is a LittlewoodRichardson multitableau of shape $\lambda, T=\left(T_{1}, \ldots, T_{r}\right)$ is a Littlewood-Richardson multitableau of shape $\mu$ and both $S_{i}$ and $T_{i}$ have the same content $\rho(i)$ for some partition $\rho(i)$ of $\pi_{i}$, for all $i \in[r]$. Let $c_{(\rho(1), \ldots, \rho(r))}^{\lambda}$ denote the number of Littlewood-Richardson multitableaux of shape $\lambda$ and content $(\rho(1), \ldots, \rho(r))$ and let $\operatorname{Ir}(\lambda, \mu ; \pi)$ denote the cardinality of $\operatorname{LR}(\lambda, \mu ; \pi)$. Then

$$
\operatorname{lr}(\lambda, \mu ; \pi)=\sum_{\rho(1) \vdash \pi_{1}, \ldots, \rho(r) \vdash \pi_{r}} c_{(\rho(1), \ldots, \rho(r))}^{\lambda} c_{(\rho(1), \ldots, \rho(r))}^{\mu} .
$$

Similar numbers have already proved to be useful in the study of minimal components, in the dominance order of partitions, of Kronecker products [14].

The number $\operatorname{Ir}(\lambda, \mu ; \pi)$ can be described as an inner product of characters. For this description we need the permutation character $\phi^{\pi}:=\operatorname{Ind}_{\mathcal{S}_{\pi}}^{S_{m}}\left(1_{\pi}\right)$, namely, the induced character from the trivial character of $\mathrm{S}_{\pi}=\mathrm{S}_{\pi_{1}} \times \cdots \times \mathrm{S}_{\pi_{r}}$. It follows from Frobenius reciprocity and the Littlewood-Richardson rule that (see also $[6,2.9 .17]$ )

4.1 Lemma. Let $\lambda, \mu, \pi$ be as above. Then

$$
\operatorname{lr}(\lambda, \mu ; \pi)=\left\langle\chi^{\lambda} \otimes \chi^{\mu}, \phi^{\pi}\right\rangle .
$$

Since Young's rule and Lemma 4.1 imply that $\operatorname{tr}(\lambda, \mu ; \nu) \geq \mathrm{k}(\lambda, \mu, \nu)$, then we have 4.2 Corollary. Let $\lambda, \mu, \nu$ be partitions of $m$. If $\operatorname{lr}(\lambda, \mu ; \nu)=0$, then $\mathrm{k}(\lambda, \mu, \nu)=0$. 
4.3 Lemma. Let $\lambda, \mu, \nu$ be partitions of $m$ of lengths $p, q, r$, respectively. If $p=q r$, and $\mu_{q}<r \lambda_{p}$ or $\nu_{r}<q \lambda_{p}$, then $\operatorname{Ir}(\lambda, \mu ; \nu)=0$.

Proof. We assume that $\operatorname{lr}(\lambda, \mu ; \nu)>0$ and show that $\mu_{q} \geq r \lambda_{p}$ and $\nu_{r} \geq q \lambda_{p}$. Let $(S, T)$ be an element in $\operatorname{LR}(\lambda, \mu ; \nu)$ having content $(\rho(1), \ldots, \rho(r))$. Since $T_{i}$ is contained in $\mu$, one has, by elementary properties of Littlewood-Richardson tableaux, that $\ell(\rho(i)) \leq \ell(\mu)=q$. For any $i$, let $n_{i}$ be the number of squares of $S_{i}$ that are in column $\lambda_{p}$ of $\lambda$, then $n_{i} \leq q$. We conclude that $p=n_{1}+\cdots+n_{r} \leq r q=p$. Therefore $n_{i}=q=\ell(\rho(i))$ for all $i$. This forces that each $S_{i}$ contains a $j$ in the squares $(j+(i-1) q, 1), \ldots,\left(j+(i-1) q, \lambda_{p}\right)$ of $\lambda$, for all $j \in[q]$. So, $\rho(i)_{j} \geq \lambda_{p}$ for all $j$. In particular, for $i=r$, since $S_{r}$ has $\nu_{r}$ squares, one has $\nu_{r} \geq q \lambda_{p}$. Now, since $\ell(\mu)=q$, all entries of $T_{i}$ equal to $q$ must be in row $q$ of $\mu$. Then $\mu_{q} \geq \rho(1)_{q}+\cdots+\rho(r)_{q} \geq r \lambda_{p}$. The claim follows.

4.4 Example. To illustrate the idea in the proof of the previous lemma let $\lambda=(8,5,4,3)$ and let $\mu$ and $\nu$ be partitions of 20 length 2 . Let $(S, T)$ be any multitableau in $\operatorname{LR}(\lambda, \mu ; \nu)$. Then, elementary properties of Littlewood-Richardson tableaux force $S$ to have the form

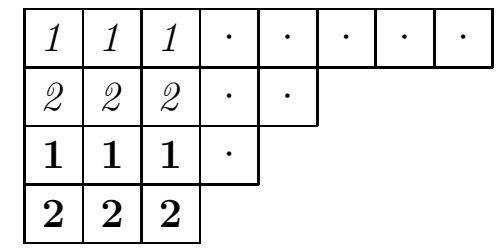

Here $S=\left(S_{1}, S_{2}\right), S_{1}$ is formed by italic numerals and $S_{2}$ by boldface numerals. The dots indicate entries that can be either in $S_{1}$ or $S_{2}$. This partial information on $S$ forces $\mu_{2} \geq 6$ and $\nu_{2} \geq 6$.

4.5 Corollary. Let $\lambda, \mu, \nu$ be partitions of $m$ of length $p, q, r$, respectively. If $p=q r$, and $\mu_{q}<r \lambda_{p}$ or $\nu_{r}<q \lambda_{p}$, then $\mathrm{k}(\lambda, \mu, \nu)=0$.

Proof. This follows from Lemma 4.3 and Corollary 4.2 .

Corollary 4.5 and Theorem 3.1 imply the following

4.6 Theorem. Let $\lambda, \mu, \nu$ be partitions of $m$ of length $p, q, r$, respectively. Let $t=\lambda_{p}$ and assume $p=q r$, then we have

(1) If $\mu_{q}<$ rt or $\nu_{r}<q$ t, then $\mathrm{k}(\lambda, \mu, \nu)=0$.

(2) If $\mu_{q} \geq r t$ and $\nu_{r} \geq q t$, let $\widetilde{\lambda}=\lambda-(t)^{p}, \widetilde{\mu}=\mu-(r t)^{q}$ and $\widetilde{\nu}=\nu-(q t)^{r}$. Then, $\mathrm{k}(\lambda, \mu, \nu)=\mathrm{k}(\widetilde{\lambda}, \widetilde{\mu}, \widetilde{\nu})$.

\section{Applications}

We conclude this paper with an application to the expansion of $\chi^{\mu} \otimes \chi^{\nu}$ when $\ell(\mu)=$ $2=\ell(\nu)$. It is well known that any component of $\chi^{\mu} \otimes \chi^{\nu}$ corresponds to a partition of length at most $\left|\mu \cap \nu^{\prime}\right| \leq 4$, see Satz 1 in [4], Theorem 1.6 in [5] or Theorem 2.1 in [9]. 
Even in this simple case a nice closed formula seems unlikely to exist. J. Remmel and T. Whitehead (Theorem 2.1 in [9]) gave a close, though intricate, formula for $\mathrm{k}(\lambda, \mu, \nu)$ valid for any $\lambda$ of length at most 4; M. Rosas (Theorem 1 in [10]) gave a formula of combinatorial nature for $\mathrm{k}(\lambda, \mu, \nu)$, which requires taking subtractions, also valid for any $\lambda$ of length at most 4; C. Ballantine and R. Orellana (Proposition 4.12 in [2]) gave a simpler formula for $\mathrm{k}(\lambda, \mu, \nu)$, at the cost of assuming an extra condition on $\lambda$.

Note that when $\ell(\lambda)=1$ the coefficient $\mathrm{k}(\lambda, \mu, \nu)$ is trivial to compute. For $\ell(\lambda)=2$ the Remmel-Whitehead formula for $\mathrm{k}(\lambda, \mu, \nu)$ reduces to a simpler one (Theorem 3.3 in [9]). This formula was recovered by Rosas in a different way (Corollary 1 in [10]). So, the nontrivial cases are those for which $\ell(\lambda)=3,4$. Corollary 5.1 deals with the case of length 4. On the one hand it gives a new vanishing condition. On the other hand, when this vanishing condition does not hold, it reduces the case of length 4 to the case of length 3. Thus, this reduction would help to simplify the proofs of the formulas given by Remmel-Whitehead and Rosas.

The following corollary is a particular case of Theorem 4.6.

5.1 Corollary. Let $\lambda, \mu, \nu$ be a partitions of $m$ of length 4, 2, 2, respectively. Let $t=\lambda_{4}$, then we have

(1) If $\mu_{2}<2 t$ or $\nu_{2}<2 t$, then $\mathrm{k}(\lambda, \mu, \nu)=0$.

(2) If $\mu_{2} \geq 2 t$ and $\nu_{2} \geq 2 t$, let $\widetilde{\lambda}=\left(\lambda_{1}-t, \lambda_{2}-t, \lambda_{3}-t\right), \widetilde{\mu}=\left(\mu_{1}-2 t, \mu_{2}-2 t\right)$ and $\widetilde{\nu}=\left(\nu_{1}-2 t, \nu_{2}-2 t\right)$. Then, $\mathrm{k}(\lambda, \mu, \nu)=\mathrm{k}(\widetilde{\lambda}, \widetilde{\mu}, \widetilde{\nu})$.

Another observation of Remmel and Whitehead (Theorems 3.1 and 3.2 in [9]) is that their formula simplifies considerably in the case $\lambda_{3}=\lambda_{4}$. Corollary 5.1 explains this phenomenon since, in this case, the computation of $\mathrm{k}(\lambda, \mu, \nu)$ reduces to the computation of a Kronecker coefficient involving only three partitions of length at most 2, which have a simple nice formula (Theorem 3.3 in [9]). In fact, combining our result with this simple formula we obtain a new one. For completeness we record here the Remmel-Whitehead formula in the equivalent version of Rosas.

In the next theorems the notation $(y \geq x)$ means 1 if $y \geq x$ and 0 if $y \nsupseteq x$.

5.2 Theorem. [9, Theorem 3.3] Let $\lambda, \mu, \nu$ be partitions of $m$ of length 2. Let $x=$ $\max \left(0,\left\lceil\frac{\nu_{2}+\mu_{2}+\lambda_{2}-m}{2}\right\rceil\right)$ and $y=\left\lceil\frac{\nu_{2}+\mu_{2}-\lambda_{2}+1}{2}\right\rceil$. Assume $\nu_{2} \leq \mu_{2} \leq \lambda_{2}$. Then

$$
\mathrm{k}(\lambda, \mu, \nu)=(y-x)(y \geq x) .
$$

From Corollary 5.1 and Theorem 5.2 we obtain

5.3 Theorem. Let $\lambda, \mu, \nu$ be partitions of $m$ of length 4, 2, 2, respectively. Suppose that $\lambda_{3}=\lambda_{4}$ and that $2 \lambda_{3} \leq \nu_{2} \leq \mu_{2}$. Let $x=\max \left(0,\left\lceil\frac{\nu_{2}+\mu_{2}+\lambda_{2}-\lambda_{3}-m}{2}\right\rceil\right), y=\left\lceil\frac{\nu_{2}+\lambda_{2}-\mu_{2}-\lambda_{3}+1}{2}\right\rceil$ and $z=\left\lceil\frac{\nu_{2}+\mu_{2}-\lambda_{2}-3 \lambda_{3}+1}{2}\right\rceil$. We have

(1) If $\lambda_{2}+\lambda_{3} \leq \mu_{2}$, then $\mathrm{k}(\lambda, \mu, \nu)=(y-x)(y \geq x)$.

(2) If $\lambda_{2}+\lambda_{3}>\mu_{2}$, then $\mathrm{k}(\lambda, \mu, \nu)=(z-x)(z \geq x)$. 
Proof. Let $\tilde{\lambda}=\left(\lambda_{1}-\lambda_{3}, \lambda_{2}-\lambda_{3}\right), \widetilde{\mu}=\left(\mu_{1}-2 \lambda_{3}, \mu_{2}-2 \lambda_{3}\right)$ and $\widetilde{\nu}=\left(\nu_{1}-2 \lambda_{3}, \nu_{2}-2 \lambda_{3}\right)$. These are partitions of $m-4 \lambda_{3}$. Then, by Corollary $5.1, \mathrm{k}(\lambda, \mu, \nu)=\mathrm{k}(\widetilde{\lambda}, \widetilde{\mu}, \widetilde{\nu})$. Since $\ell(\widetilde{\lambda})=\ell(\widetilde{\mu})=\ell(\widetilde{\nu})=2$, we can apply Theorem 5.2. Due to the symmetry of the Kronecker coefficients we are assuming $\nu_{2} \leq \mu_{2}$. We have to consider three cases: (a) $\lambda_{2}-\lambda_{3} \leq \nu_{2}-2 \lambda_{3}$, (b) $\nu_{2}-2 \lambda_{3}<\lambda_{2}-\lambda_{3} \leq \mu_{2}-2 \lambda_{3}$ and (c) $\mu_{2}-2 \lambda_{3}<\lambda_{2}-\lambda_{3}$. In the first two cases the Remmel-Whitehead formula yields the same formula for $\mathrm{k}(\widetilde{\lambda}, \widetilde{\mu}, \widetilde{\nu})$. So, we have only two cases to consider: (1) $\lambda_{2}+\lambda_{3} \leq \mu_{2}$ and (2) $\mu_{2}<\lambda_{2}+\lambda_{3}$. In the first case Theorem 5.2 yields

$$
\mathrm{k}(\widetilde{\lambda}, \widetilde{\mu}, \widetilde{\nu})=\left(y^{\prime}-x^{\prime}\right)\left(y^{\prime} \geq x^{\prime}\right)
$$

where $x^{\prime}=\max \left(0,\left\lceil\frac{\nu_{2}-2 \lambda_{3}+\lambda_{2}-\lambda_{3}+\mu_{2}-2 \lambda_{3}-\left(m-4 \lambda_{3}\right)}{2}\right\rceil\right)$ and $y^{\prime}=\left\lceil\frac{\nu_{2}-2 \lambda_{3}+\lambda_{2}-\lambda_{3}-\left(\mu_{2}-2 \lambda_{3}\right)+1}{2}\right\rceil$. It is straightforward to check that $x^{\prime}=x$ and $y^{\prime}=y$, so the first claim follows.

The second case is similar.

\section{References}

[1] C.M. Ballantine and R.C. Orellana, On the Kronecker product $s(n-p, p) * s_{\lambda}$, Electron. J. Combin. 12 (2005) Reseach Paper 28, 26 pp. (electronic).

[2] C.M. Ballantine and R.C. Orellana, A combinatorial interpretation for the coefficients in the Kronecker product $s(n-p, p) * s_{\lambda}$, Sém. Lotar. Combin. 54A (2006), Art. B54Af, 29pp. (electronic).

[3] M. Brion, Stable properties of plethysm: on two conjectures of Foulkes, manuscripta math. 80 (1993), 347-371.

[4] M. Clausen and H. Meier, Extreme irreduzible Konstituenten in Tensordarstellungen symmetrischer Gruppen, Bayreuther Math. Schriften 45 (1993), 1-17.

[5] Y. Dvir, On the Kronecker product of $S_{n}$ characters, J. Algebra 154 (1993), 125-140.

[6] G.D. James and A. Kerber, "The representation theory of the symmetric group", Encyclopedia of mathematics and its applications, Vol. 16, Addison-Wesley, Reading, Massachusetts, 1981.

[7] I.G. Macdonald, "Symmetric functions and Hall polynomials," 2nd. edition Oxford Mathematical Monographs Oxford Univ. Press 1995.

[8] F.D. Murnaghan, The analysis of the Kronecker product of irreducible representations of the symmetric group, Amer. J. Math. 60 (1938), 761-784.

[9] J.B. Remmel and T. Whitehead, On the Kronecker product of Schur functions of two row shapes, Bull. Belg. Math. Soc. 1 (1994), 649-683.

[10] M.H. Rosas, The Kronecker product of Schur functions indexed by two-row shapes or hook shapes, J. Algebraic Combin. 14 (2001), 153-173.

[11] B. Sagan, "The symmetric group. Representations, combinatorial algorithms and symmetric functions". Second ed. Graduate Texts in Mathematics 203. Springer Verlag, 2001. 
[12] R.P. Stanley, "Enumerative Combinatorics, Vol. 2" , Cambridge Studies in Advanced Mathematics 62. Cambridge Univ. Press, 1999.

[13] E. Vallejo, Stability of Kronecker product of irreducible characters of the symmetric group, Electron. J. Combin 6 (1999) Reseach Paper 39, 7 pp. (electronic).

[14] E. Vallejo, Plane partitions and characters of the symmetric group, J. Algebraic Combin. 11 (2000), 79-88. 